\title{
Efecto de la administración oral de zinc sobre sensibilidad a la insulina y niveles séricos de leptina y andrógenos en hombres con obesidad
}

\author{
Anel Gómez-García1,2,3,a, Eduardo Hernández-Salazar2,a, \\ Manuel González-O rtiz 2,a,b, Esperanza Martínez-Abundis²,a,b. \\ Effect of oral zinc administration \\ on insulin sensitivity, \\ leptin and androgens in obese males
}

Background: Zinc is important for insulin synthesis, storage and secretion. When zinc concentration decrease, there is a concomitant reduction in insulin secretion and peripheral insulin sensitivity Aim: To assess the effects of zinc sulfate on insulin sensitivity, leptin and androgens in obese individuals. Material and methods: A randomized, double-blind, placebo-controlled clinical trial was performed in 14 obese volunteers between 21 and 30 years old, with body mass index (BMI) $\geq 27 \mathrm{~kg} / \mathrm{m} 2$. During one month, seven subjects received $100 \mathrm{mg} /$ day of zinc sulfate orally $(\mathrm{ZnG})$ and the other seven received placebo, as control group (CG). At baseline and after the intervention, insulin sensitivity was measured using a euglycemic-hyperinsulinemic clamp technique. Blood glucose, serum lipids, zinc, androgens and leptin were also measured in a fasting blood sample. Results: After the intervention, a rise in zinc concentrations from 11.8 to 16.9 umol/L; $p=0.001$ and in leptin levels from 15.2 to $27.7 \mathrm{ng} / \mathrm{mL} ; \mathrm{p}=0.029$, was observed in the $\mathrm{ZnG}$. No changes were observed in the CG. There were no significant changes in insulin sensitivity and androgens after the intervention with zinc sulfate. Conclusions: Zinc increased the leptin concentrations in obese individuals, but did not modify insulin sensitivity and androgens (Rev Méd Chile 2006; 134: 279-84).

(Key words: Hyperinsulinism; Insulin; Leptin.

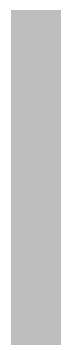

\footnotetext{
Recibido el 4 de agosto, 2004. Aceptado el 22 de agosto, 2005.

Trabajo financiado en forma parcial por el Fondo de Fomento a la Investigación (FOFOI) del Instituto Mexicano del Seguro Social.

${ }^{1}$ Centro Universitario de Ciencias de la Salud, Universidad de Guadalajara. ${ }^{2}$ Unidad de Investigación Médica en Epidemiología Clínica, Unidad Médica de Alta Especialidad, Hospital de Especialidades. Centro Médico Nacional de Occidente, Instituto Mexicano del Seguro Social. ${ }^{3}$ Centro de Investigación Biomédica de Michoacán, Instituto Mexicano del Seguro Social.

${ }^{\mathrm{a}}$ Maestro en Ciencias

${ }^{\mathrm{b}}$ Doctor en Ciencias
}

Correspondencia a: Dra. en C. Esperanza Martínez Abundis. Montes Urales 1409, Colonia Independencia. 44340. Guadalajara, México. Tel. +52 (33) 38267022. Fax: +52 (33) 36161218. E mail: uiec@prodigy.net.mx 
$\mathrm{L}$ a relación entre la insulina y el zinc se conoce desde hace varias décadas. El zinc es importante para la síntesis, almacenamiento y secreción de insulina: la disminución en las concentraciones de zinc se asocia con una reducción en la secreción de insulina, con una resistencia tisular a la acción de esta hormona ${ }^{1-3} \mathrm{y}$, consecuentemente, con un incremento en la glucosa circulante ${ }^{4}$. Estudios in vitro demuestran que a bajas concentraciones de zinc existe estimulación de la gluconeogénesis e inhibición de la glucólisis ${ }^{5}$. La literatura muestra controversia acerca de las concentraciones de zinc sérico en sujetos con obesidad, ya que unos estudios reportan disminución ${ }^{6,7}$ y otros aumento ${ }^{8,9}$ de este elemento.

La obesidad en el hombre se asocia con un gran número de anormalidades metabólicas que incluyen resistencia a la insulina, hiperinsulinemia, dislipidemia, hiperleptinemia con resistencia a la leptina, disminución en las concentraciones de la globulina fijadora de hormonas sexuales (SHBG), testosterona total (TT) y testosterona libre (TL) ${ }^{10-14}$. Los sujetos con obesidad que cursan con aumento en las concentraciones de leptina, presentan disminución en la SHBG y en la $\mathrm{TT}^{15}$. No se conoce la participación del zinc sobre la acción de la insulina y su relación con la SHBG, TT, TL y leptina. El objetivo del presente estudio fue identificar el efecto de una intervención farmacológica con sulfato de zinc sobre la sensibilidad a la insulina, la leptina y los andrógenos en hombres con obesidad.

\section{Material y MÉTOdos}

Se llevó a cabo un ensayo clínico al azar, doble ciego, controlado con placebo, en 14 hombres entre 21 y 30 años de edad, con obesidad [índice de masa corporal (IMC) $\geq 27 \mathrm{~kg} / \mathrm{m}^{2}$ ]. Los voluntarios tenían familiares en primer grado de diabetes mellitus tipo 2 y cursaron con un peso corporal estable al menos tres meses antes del estudio. A todos los sujetos se les efectuó una historia clínica y nutricional. Se incluyeron aquellos con tensión arterial $<140 / 90 \mathrm{mmHg}$, glucosa sérica en ayuno $<100 \mathrm{mg} / \mathrm{dL}$, sin dislipidemia, ni alteraciones en el ácido úrico y en la creatinina. No ingirieron ningún medicamento que alterara el metabolismo de carbohidratos, lípidos, zinc u hormonas.
La aleatorización de los participantes para recibir sulfato de zinc o placebo se realizó mediante el azar simple, con la selección de un sobre cerrado que contenía la opción A o B. Ni el investigador, ni los participantes conocieron durante el estudio el tipo de intervención. Al final del estudio se constituyeron 2 grupos: un grupo de 7 voluntarios que ingirió vía oral una cápsula de $100 \mathrm{mg}$ de sulfato de zinc (Mallinckrodt Backer, Inc. Paris), cada $24 \mathrm{~h}$, durante un período de 30 días (GZn) y un grupo de 7 individuos que ingirió una cápsula con 100 mg de placebo al día, de características similares al suplemento y durante el mismo tiempo, que sirvió como grupo control (GC). La adherencia al tratamiento se supervisó con la contabilización de las cápsulas sobrantes cada semana.

A cada sujeto se le realizó al inicio y al final de la intervención farmacológica, previo ayuno de 12 h, una pinza euglucémica-hiperinsulinémica para estimar la sensibilidad a la insulina, la cuantificación sérica de zinc, leptina, insulina, TT, TL, SHBG y un perfil bioquímico [glucosa, colesterol total, triglicéridos, lipoproteínas de alta densidad (HDL), ácido úrico y creatinina].

La sensibilidad a la insulina correspondió al valor del metabolismo total de la glucosa (M) obtenido mediante el método de la pinza euglucémica-hiperinsulinémica, descrita previamente por De Fronzo y cols ${ }^{16}$. Para la realización de esta prueba se instalaron dos accesos venosos, el primero en forma retrógrada en cualquier vena de la mano, por medio de un catéter $23 \mathrm{G}$, para la toma de muestras a lo largo de la prueba; la mano se envolvió en un colchón térmico con el fin de alcanzar una temperatura local superior a $40^{\circ} \mathrm{C}$ para arterializar la sangre. El segundo acceso venoso se instaló en el brazo contralateral con un catéter $23 \mathrm{G}$ como vía de infusión. La insulina (Humulin R, Eli Lilly, Co. México) se infundió en forma continua, con el objetivo de alcanzar valores de insulina en sangre de $\sim 100 \mu \mathrm{U} / \mathrm{mL}$, por lo que la tasa de infusión de insulina se fijó a $40 \mu \mathrm{U} / \mathrm{m}^{2}$.min. La concentración de glucosa sanguínea se mantuvo constante (aproximadamente a $90 \mathrm{mg} / \mathrm{dL}$, con un coeficiente de variación $<5 \%$ ) a lo largo del estudio (120 min), mediante una infusión de glucosa al 10\% a tasa variable, de acuerdo a las determinaciones de glucosa sanguínea llevadas a cabo en intervalos de 5 min. Al final de los $120 \mathrm{~min}$ de la prueba de 
pinza, se mantuvo la infusión de glucosa al 10\% por 30 min más, como precaución para evitar hipoglucemia en el voluntario.

El zinc sérico se cuantificó por espectrometría de absorción atómica. La glucosa se determinó por la técnica de glucosa-oxidasa y los lípidos (colesterol total, colesterol-HDL y triglicéridos), creatinina y ácido úrico séricos, por métodos enzimáticos colorimétricos en equipo automatizado (Dimension Chemistry System, Dade Behring). El colesterol de las lipoproteínas de baja densidad (LDL) se estimó mediante la fórmula de Friedewald (colesterol-LDL $=$ colesterol total - colesterol-HDL $-\mathrm{TG} / 5)$ y las lipoproteínas de muy baja densidad (VLDL) se calcularon como triglicéridos/5. La insulina, TT, TL y SHBG se cuantificaron por radioinmunoanálisis y la leptina mediante IRMA (Diagnostic Products Corporation, Los Angeles, California, USA). Cada determinación con coeficientes de variación intra- e inter-ensayo menores al 3 y $5 \%$, respectivamente.

El tamaño de la muestra se calculó con una fórmula para ensayos clínicos ${ }^{17}$ con un nivel de confianza de 95\%, una potencia de la prueba de $80 \%$, una desviación estándar de la $\mathrm{M}$ de la pinza euglucémica-hiperinsulinémica de $0,8 \mathrm{mg} / \mathrm{kg}$.min y una diferencia esperada de, al menos, el doble de la desviación estándar (1,6 mg/kg.min), con lo que se obtuvo un resultado de 6 pacientes por grupo. Se incluyeron 7 pacientes por grupo por la posibilidad de pérdidas durante el seguimiento. La prueba de Wilcoxon se utilizó para el análisis entre, antes y después de la intervención farmacológica. La pueba U de Mann-Whitney se usó para comparar los cambios entre ambos grupos. Se consideró un nivel de significación a un valor de $\mathrm{p}<0,05$.

Con el fin de contar con un valor de referencia de las concentraciones séricas de zinc en individuos sin obesidad (IMC $<27 \mathrm{~kg} / \mathrm{m}^{2}$ ), que sirviera de comparación, se realizó su determinación en 15 hombres de 21 a 30 años de edad, que negaron el antecedente familiar en primer grado de diabetes mellitus tipo 2 e hipertensión arterial y a los cuales no se les practicó el resto de determinaciones de laboratorio contempladas en el estudio para los sujetos con obesidad.

Los participantes firmaron una hoja de consentimiento bajo información, el estudio reunió requisitos para realizar investigación en seres humanos y fue aprobado por el Comité de Investigación y Ética hospitalario.

\section{Resultados}

La edad de los voluntarios fue de 21,8 $\pm 2,8$ y $25,1 \pm 4,5$ años para el GZn y el GC, respectivamente; $(\mathrm{p}=0,318)$. Ambos grupos tuvieron un IMC similar $\left(30,7 \pm 2,6\right.$ vs $30,5 \pm 3,9 \mathrm{~kg} / \mathrm{m}^{2} ; \mathrm{p}=0,710$, para el GZn y el GC, respectivamente).

Se encontró una concentración menor de zinc sérico en ambos grupos $[11,8(8,3-13,7)$ para el GZn y $12,9(7,7-17,4) \mu \mathrm{mol} / \mathrm{L}$ para el GC], en comparación con el valor de 21,8 (15,9-26,7) $\mu \mathrm{mol} / \mathrm{L}$ que obtuvimos en la población de varones jóvenes $(24,3 \pm 2,7$ años), no obesos (IMC de $23,1 \pm 1,2 \mathrm{~kg} / \mathrm{m}^{2}$ ).

La sensibilidad a la insulina, estimada por el valor M obtenido por la técnica de pinza euglucémica-hiperinsulinémica, fue similar en ambos grupos de estudio antes de la intervención farmacológica al igual que la leptina, los andrógenos y el resto del perfil bioquímico (Tabla 1).

Después de administrar un suplemento de sulfato de zinc, se encontró un incremento en las concentraciones séricas de zinc (Figura 1) y de leptina (Figura 2). La TL mostró una tendencia a aumentar después de la intervención farmacológica con sulfato de zinc $(9,8 \pm 2,3$ vs $12,3 \pm 4,7 \mathrm{nmol} / \mathrm{L}$; $\mathrm{p}=0,082$ ). La sensibilidad a la insulina y el perfil bioquímico no se modificaron con la administración de sulfato de zinc (ver valores en la Tabla 1). El placebo no modificó la sensibilidad a la insulina, la leptina, los andrógenos, ni el perfil bioquímico de los voluntarios (ver valores en la Tabla 1). El IMC no fue diferente entre los dos grupos al final de la intervención $(31,3 \pm 2,6$ vs $30,3 \pm 4,3 \mathrm{~kg} / \mathrm{m}^{2}$ para el GZn y el GC, respectivamente; $\mathrm{p}=0,535$ ).

La adherencia, tanto para el suplemento como para el placebo, fue superior a $95 \%$. El sulfato de zinc y el placebo fueron bien tolerados y los voluntarios no presentaron efectos indeseables a lo largo del estudio.

\section{Discusión}

La obesidad se asocia con un número de anormalidades metabólicas y hormonales que incluyen resistencia a la insulina, dislipidemia, disminución en las concentraciones de TT, TL y $\mathrm{SHBG}^{18}$. Además, existe evidencia en modelos animales de 
Tabla 1. Sensibilidad a la insulina, leptina, andrógenos y perfil bioquímico en los grupos de estudio

\begin{tabular}{|lcccccc|}
\hline & \multicolumn{3}{c}{ Antes de la Intervención } & \multicolumn{4}{c|}{ Después de la Intervención } \\
& GZn & $\mathrm{GC}$ & $\mathrm{p}$ & $\mathrm{GZn}$ & $\mathrm{GC}$ & $\mathrm{p}$ \\
& $\mathrm{n}=7$ & $\mathrm{n}=7$ & & $\mathrm{n}=7$ & $\mathrm{n}=7$ & \\
\hline Insulina, $(\mu \mathrm{U} / \mathrm{mL})$ & $13,0 \pm 11,0$ & $6,7 \pm 2,8$ & 0,091 & $10,5 \pm 4,9$ & $10,1 \pm 5,5$ & 0,805 \\
$\mathrm{M},(\mathrm{mg} / \mathrm{kg} . \mathrm{min})$ & $3,6 \pm 0,9$ & $4,0 \pm 2,0$ & 0,902 & $3,6 \pm 1,0$ & $3,8 \pm 1,5$ & 0,902 \\
Glucosa, $(\mathrm{mg} / \mathrm{dL})$ & $94,6 \pm 4,6$ & $80,8 \pm 9,0$ & 0,128 & $91,5 \pm 7,5$ & $90,0 \pm 9,14$ & 0,165 \\
Colesterol total, (mg/dL) & $155,8 \pm 15,4$ & $163,9 \pm 31,7$ & 0,165 & $151,4 \pm 16,7$ & $163,2 \pm 33,1$ & 0,128 \\
HDL, $(\mathrm{mg} / \mathrm{dL})$ & $35,1 \pm 4,6$ & $37,8 \pm 7,7$ & 0,152 & $35,1 \pm 6,4$ & $42,8 \pm 8,8$ & 0,097 \\
LDL, $(\mathrm{mg} / \mathrm{dL})$ & $91,2 \pm 13,1$ & $109,0 \pm 43,3$ & 0,165 & $91,6 \pm 16,9$ & $99,8 \pm 41,6$ & 0,383 \\
Triglicéridos, (mg/dL) & $145,5 \pm 32,2$ & $131,7 \pm 41,2$ & 0,710 & $123,4 \pm 30,2$ & $132,5 \pm 32,1$ & 0,620 \\
VLDL, (mg/dL) & $29,1 \pm 6,4$ & $33,4 \pm 22,2$ & 0,902 & $24,6 \pm 6,0$ & $31,4 \pm 11,1$ & 0,259 \\
Creatinina, $(\mathrm{mg} / \mathrm{dL})$ & $0,9 \pm 0,1$ & $0,9 \pm 0,1$ & 0,915 & $0,8 \pm 0,1$ & $0,9 \pm 0,1$ & 0,805 \\
Acido úrico, $(\mathrm{mg} / \mathrm{dL})$ & $6,3 \pm 0,7$ & $6,9 \pm 1,7$ & 0,456 & $6,0 \pm 0,8$ & $6,4 \pm 0,7$ & 0,535 \\
Leptina, $(\mathrm{ng} / \mathrm{mL})$ & $16,4 \pm 7,1$ & $17,7 \pm 6,3$ & 0,817 & $20,2 \pm 7,9$ & $18,1 \pm 5,5$ & 0,537 \\
TT, $(\mathrm{ng} / \mathrm{mL})$ & $5,2 \pm 1,5$ & $5,1 \pm 2,5$ & 0,620 & $6,0 \pm 2,1$ & $4,5 \pm 2,6$ & 0,128 \\
TL, $(\mathrm{ng} / \mathrm{mL})$ & $9,8 \pm 2,3$ & $6,3 \pm 2,4$ & 0,535 & $12,3 \pm 4,7$ & $5,7 \pm 2,8$ & 0,011 \\
SHBG, $(\mathrm{ng} / \mathrm{mL})$ & $23,4 \pm 11,1$ & $37,4 \pm 6,9$ & 0,710 & $26,8 \pm 12,2$ & $33,8 \pm 11,1$ & 0,259 \\
\hline
\end{tabular}

M= Metabolismo total de la glucosa que estima la sensibilidad a la insulina. HDL= Lipoproteínas de alta densidad. LDL= Lipoproteínas de baja densidad. VLDL= Lipoproteínas de muy baja densidad. TT=Testosterona total. TL=Testosterona libre. SHBG=globulina fijadora de hormonas sexuales.

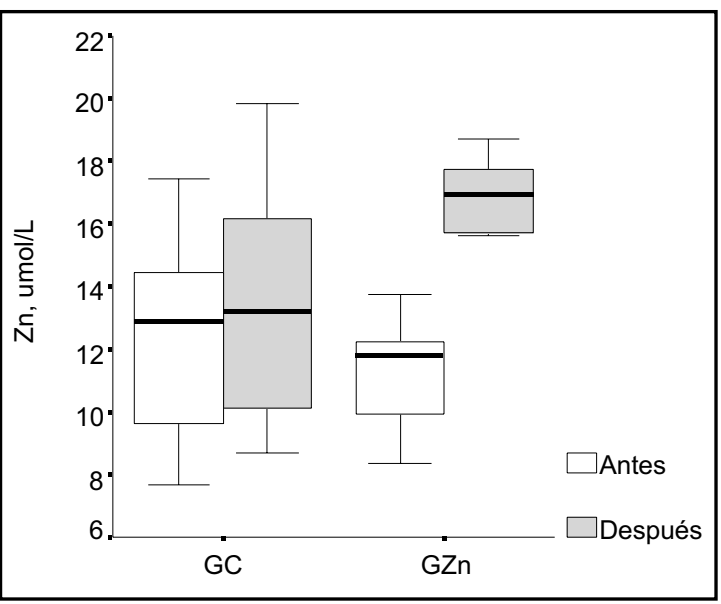

Figura 1. Concentraciones séricas de zinc antes y después de la intervención en el grupo control (GC) $[12,9(7,7-17,4)$ vs $13,2(8,7-19,9) \mu \mathrm{mol} / \mathrm{L}] ; \mathrm{p}=0,310 \mathrm{y}$ en el grupo con sulfato de zinc (GZn) $[11,8(8,3-13,7)$ vs $16,9(15,6-18,7) \mu \mathrm{mol} / \mathrm{L}] ; \mathrm{p}=0,0001$, en hombres con obesidad.

*Prueba de Wilcoxon.

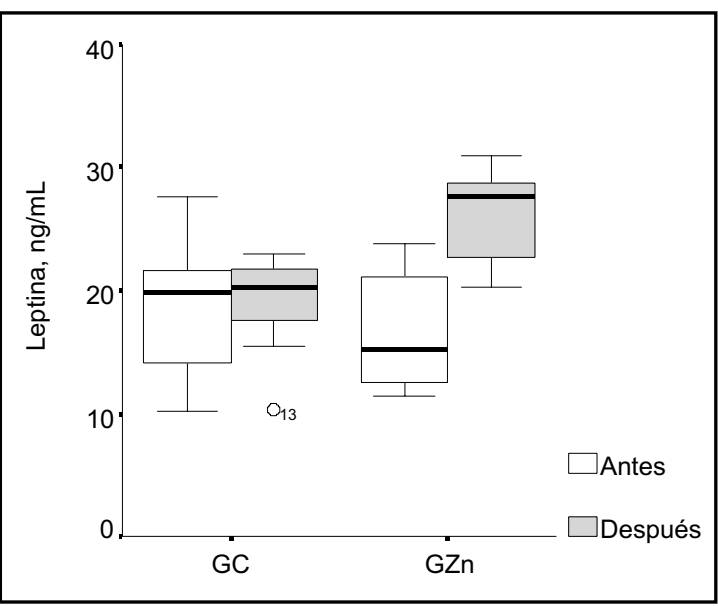

Figura 2. Concentraciones séricas de leptina antes y después de la intervención farmacológica en el grupo control (GC) [20,3 (10,2-27,6) vs 20,2 (10,3$23,0) \mathrm{ng} / \mathrm{mL}$; $\mathrm{p}=0,612$ y en el grupo con sulfato de Zinc (GZn) [15,2 (20,3-30,9) vs $27,7(20,3-30,9) \mathrm{ng} /$ $\mathrm{mL}$; $\mathrm{p}=0,029$, en hombres con obesidad.

*Prueba de Wilcoxon. 
otras alteraciones relacionadas con la obesidad como sería una disminución en las concentraciones de zinc ${ }^{19}$. El zinc es uno de los elementos más importantes en la nutrición y en la salud de los humanos, pues desempeña un papel importante en una serie de procesos metabólicos como la síntesis, almacenamiento y secreción de la insulina, además, juega un papel importante en la acción de la leptina y de los andrógenos ${ }^{8}$. Nuestro estudio demostró que los sujetos jóvenes con obesidad presentaron bajas concentraciones de zinc, hallazgo similar a lo que ocurre en ratas con obesidad inducida de forma genética o con $\operatorname{dieta}^{19}$. La disminución en cualquier elemento traza se puede deber a una reducción en la ingesta, a un aumento en las necesidades del organismo o bien, a alteraciones en la absorción del elemento en presencia de una ingesta adecuada en la dieta ${ }^{20}$. Sin embargo, en nuestro estudio, aunque se encontró una disminución del zinc en los individuos con obesidad, esto no se asoció con modificaciones en la insulina, ni en la sensibilidad a la misma.

Se conoce que el zinc juega un papel importante en la regulación del apetito y del gasto energético; dicha regulación podría ser mediada a través de la hormona del apetito llamada lepti$n^{21,22}$. En el presente estudio se demostró que después de la intervención farmacológica con zinc en individuos obesos, la leptina incrementó significativamente sus concentraciones circulantes, sin haberse documentado una modificación del IMC.

\section{REFERENCIAS}

1. Chen MD, Lin PY, Chen PS, Cheng V, Lin WH. Zinc attenuation of GDP binding to brown adipocytes mitochondria is genetically obese (ob/ob) mice. Biol Trace Elem Res 1997; 57: 139-45.

2. Hendricks DG, Mahoney AW. Glucose tolerance in zinc deficient rats. J Nutr 1972; 102: 1079-84.

3. Huber AM, Gershoff SN. Effect of zinc deficiency in rats on insulin release from the pancreas. J Nutr 1973; 103: 1739-44.

4. Chausmer AB. Zinc, Insulin and Diabetes. J Am Coll Nutr 1998; 17: 109-15.
Se requiere de estudios posteriores para dilucidar las vías fisiopatológicas por las cuales el zinc pudiera regular apetito y gasto energético a través de la leptina.

La obesidad suele cursar con concentraciones bajas de TT y TL y una de las acciones del zinc es la modulación de los andrógenos en el hombre ${ }^{23}$. En nuestro estudio sólo se encontró una tendencia al incremento de la TL con la administración del zinc, sin modificación de la TT. Esta ausencia del aumento de los andrógenos se pudo haber debido al efecto supresor de la leptina sobre la TT y la $\mathrm{TL}^{24,25}$ y a la disminución del efecto estimulante de la insulina sobre dichas hormonas ${ }^{13}$.

Es de señalar que el no haber encontrado cambios significativos con la administración del zinc en algunas de las variables de laboratorio estudiadas, pudo ser debido a que el tamaño de la muestra fue calculado sólo para ver resultados en la sensibilidad a la insulina estimada con la $\mathrm{M}$ de la pinza euglucémica-hiperinsulinémica, por lo que esto debe tomarse como una limitación del estudio.

En conclusión, nuestro estudio demostró una baja concentración sérica de zinc en sujetos jóvenes con obesidad y la administración de sulfato de zinc, durante un mes, incrementó las concentraciones de leptina en dichos individuos, sin modificar la sensibilidad a la insulina, ni los andrógenos. Son necesarios más estudios in vitro e in vivo para identificar los posibles mecanismos del zinc que expliquen esos hallazgos.

5. BRAND IA, KieINEKE J. Intracellular zinc movement and its effect on the carbohydrate metabolism of isolated rat hepatocytes. J Biol Chem 1996; 271: 1941-9.

6. Strain J. Disturbances of micronutrient and antioxidant status in diabetes. Proc Nutr Soc 1991; 50: 591-604.

7. Faure P, Benhamou Py, Perard A, Halimi S, Roussel AM. Lipid peroxidation in insulin-dependent diabetic patients with early retina degenerative lesions: effects of an oral zinc supplementation. Eur J Clin Nutr 1995; 49: 282-8.

8. Groff JL, Gropper SS. Microminerals. In: Groff JL, Gropper SS, eds. Advanced nutrition and human 
metabolism. $3^{\text {th }}$ ed. Belmont CA: Wadsworth/ Thomson Learning, 2000; 401-69.

9. Hunt J, Matthys L, Johnson L. Zinc absorption, mineral balance, and blood lipids in women consuming controlled lactoovovegetarian and omnivorous diets for 8 wk. Am J Clin Nutr 1998; 67: 421-30.

10. Cefalu WT. Insulin Resistance: Cellular and Clinical Concepts. Proc Soc Exp Biol Med 2001; 226: 13-26.

11. Zumoff B, Strain GW, Miller LK, Rosner W, Senie R, SERES DS et AL. Plasma-free and non-sex-hormonebinding-globulin-bound testosterone are decreased in obese men in proportion to their degree of obesity. J Clin Endocrinol Metab 1990; 71: 929-31.

12. Abate N, Haffner SM, Garg A, Peshock RM, Grundy SM. Sex steroid hormones, upper body obesity, and insulin resistance. J Clin Endocrinol Metab 2002; 87: 4522-7.

13. Pasquali R, Casimirri F, De Lasio R, Mesini P, Boschi $\mathrm{S}$, Chierici $\mathrm{R}$ et aL. Insulin regulates testosterone and sex hormone-binding globulin concentrations in adult normal weight and obese men. J Clin Endocrinol Metab 1995; 80: 654-8.

14. Tsai E, Matsumoto A, Fujimoto W, Boyko E. Association between low testosterone and insulin resistance, independent of sex-hormone binding globulin: influence of body fat distribution. Diabetes 2003; 52 (Suppl 1): A231.

15. Castrogiovanni D, Perello M, Gaillard RC, Spinedi E. Modulatory role of testosterone in plasma leptin turnover in rats. Endocrine 2003; 22: 203-10.

16. De Fronzo R, Tobin JD, Andres R. Glucose clamp technique: a method for quantifying insulin secretion and resistance. Am J Physiol 1979; 237: E214-E223.
17. JeyaseElan L, RaO PSS. Methods of determining sample sizes in clinical trials. Indian Pedriatr 1989; 26: 115-21.

18. Bessesen D. Obesity as a factor [contributing factors to insulin resistance as IIsi North America Workshop: Project Committee on Insulin Resistance]. Nutr Rev 2000; 58: S12-S15.

19. Chen MD, Lin PY, Lin WH. Investigation of the relationships between zinc and obesity. Kao Hsuing I Hsueh Ko Hsueh Tsa Chih [Abstract] 1991; 7: 628-34.

20. Rosado JL. Deficiencia de zinc y sus implicaciones funcionales. Salud Pública Mex 1998; 40: 181-8.

21. Prasad AS. Zinc: the biology and therapeutics of an ion. Ann Inter Med 1996; 125: 142-4.

22. Mantzoros CS, Prasad AS, Beck FWJ, Grabowski S, Kaplan J, Adair C et al. Zinc may regulate serum leptin concentrations in humans. J Am Coll Nutr 1998; 17: 270-5.

23. Prasad AS, Mantzoros CS, Beck FWJ, Ress JW, Brewer GJ. Zinc status and serum testosterone levels of healthy adults. Nutrition 1996; 12 : 344-8.

24. Tsai EC, Matsumoto AM, Fujimoto WY, Boyko EJ. Association of bioavailable, free, and total testosterone with insulin resistance: influence of sex hormone-binding globulin and body fat. Diabetes Care 2004; 27: 861-8.

25. Laaksonen DE, Niskanen L, Punnonen K, Nyyssönen K, Tuomainen TP, Valkonen VP et al. Testosterone and sex hormone-binding globulin predict the metabolic syndrome and diabetes in middle-aged men. Diabetes Care 2004; 27: 1036-41. 